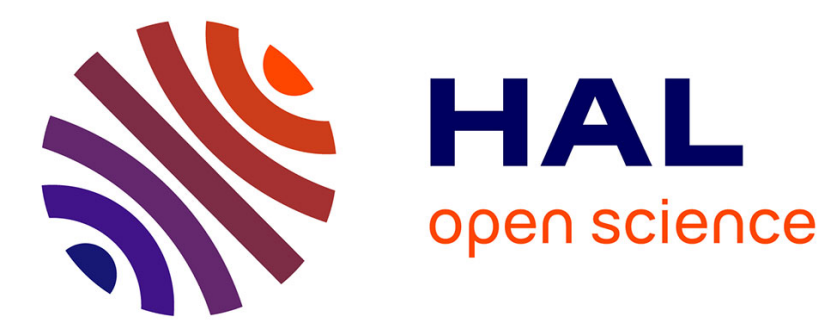

\title{
On a generalization of the Lebesgue's constant
}

Ana Alonso Rodríguez, Francesca Rapetti

\section{To cite this version:}

Ana Alonso Rodríguez, Francesca Rapetti. On a generalization of the Lebesgue's constant. Journal of Computational Physics, 2020, 10.1016/j.jcp.2020.109964 . hal-02946321

\section{HAL Id: hal-02946321 \\ https://hal.science/hal-02946321}

Submitted on 8 Oct 2020

HAL is a multi-disciplinary open access archive for the deposit and dissemination of scientific research documents, whether they are published or not. The documents may come from teaching and research institutions in France or abroad, or from public or private research centers.
L'archive ouverte pluridisciplinaire HAL, est destinée au dépôt et à la diffusion de documents scientifiques de niveau recherche, publiés ou non, émanant des établissements d'enseignement et de recherche français ou étrangers, des laboratoires publics ou privés. 


\title{
On a generalization of the Lebesgue's constant *
}

\author{
Ana Alonso Rodríguez ${ }^{\mathrm{a}}$, Francesca Rapetti ${ }^{\mathrm{b}}$ \\ ${ }^{a}$ Dip. di Matematica, Università degli Studi di Trento, 38123 Località Povo, Trento, Italy \\ ${ }^{b}$ Université Côte d'Azur, Inria, CNRS, LJAD, Parc Valrose, 06108 Nice Cedex 02, France
}

\begin{abstract}
In this work we generalize the definition of the Lebesgue's constant to the case of field interpolation by high-order Whitney's forms on simplices. We underline the important theoretical concepts at play.
\end{abstract}

Keywords: High-order Whitney's forms, weights on subsimplices, Lebesgue's constant, Vandermonde's matrix

2020 MSC: 65N30, 65D05

\section{Introduction}

The polynomial interpolation of a field $f$ over a domain $\Omega \subset \mathbb{R}^{d}, d \geq 1$, consists in the construction of a polynomial of degree $r \geq 0$ which has a finite number of degrees of freedom (dofs) concident with those of $f$. This problem is

5 a classical topic in approximation theory and matters because the high quality polynomial interpolation of fields plays an essential role in finite elements (FEs). Standard dofs for the interpolation of a scalar field $f$ in $\Omega$ are the values of $f$ at some chosen nodes in the domain and the quality of this approximaton is evaluated on the basis of the well-known Lebesgue's constant associated with 10 the set of nodes (see, e.g, [5], [7]). In many areas of physics, FEs should be able to represent vector fields from a finite number of physical coefficients, the nature of which, fluxes, circulations, etc., associate them with geometric mesh objects other than nodes 4. In electromagnetism, for example, fields are invisible and forces act without contact. The perturbations, caused to the surrounding by the 15 presence of the field, are observed via quantities, such as electromotive forces, intensities, etc., which correspond to weights on manifolds, namely line integrals (circulations), surface integrals (fluxes), etc., other than simply pointwise evaluations. The entity of physical significance is thus the $k$-form, i.e. the mapping curve $\rightarrow$ circulation $(k=1)$, surface $\rightarrow$ flux $(k=2)$, etc., and not the field itself, i.e. the electric field, the magnetic induction, etc., as a vector. The form $w$

${ }^{\star}$ Finantial support from French MathIT program ANR-15-IDEX-01 and Italian PRIN'17 project NA-FROM-PDEs.

Email addresses: ana.alonso@unitn.it (Ana Alonso Rodríguez), Francesca.Rapetti@univ-cotedazur.fr (Francesca Rapetti) 
models the field; manifolds $S$ are probes that one locates in $\Omega$ to measure the field. The value of the mesure, $\int_{S} w$, is the weight of the form $w$ on the manifold $S$. In FEs, these weights can be considered as dofs. They reflect the nature (and, if properly chosen, global regularity properties) of the field they represent and are the right objects to produce accurate high-order interpolation formulae. They are the key to extend the concepts of Lebesgue's function and constant to the interpolation of any field over a domain $\Omega$. This generalization is a novelty, resulting from adopting in FEs a point of view typical of differential geometry, thus weights as dofs.

\section{The classical framework}

Let $T$ be a $d$-simplex of $\mathbb{R}^{d}, \mathcal{P}_{r}(T)$ the space of polynomials of degree $r$ in $T$ and $N_{r}=\left(\begin{array}{c}d+r \\ d\end{array}\right)=\operatorname{dim}\left(\mathcal{P}_{r}(T)\right)$. Given $X_{r}(T)=\left\{\mathbf{x}_{1}, \ldots, \mathbf{x}_{N_{r}}\right\}$, a set of $N_{r}$ distinct nodes in $T$, the Lagrange interpolation problem for a given scalar function $f \in C^{0}(T)$ consists in finding a polynomial $\mathcal{I}_{r} f \in \mathcal{P}_{r}(T)$, such that

$$
\mathcal{I}_{r} f\left(\mathbf{x}_{i}\right)=f\left(\mathbf{x}_{i}\right), \quad \forall i=1, \ldots, N_{r} .
$$

35 Chosen a basis $\mathcal{B}=\left\{\psi_{j}\right\}_{j=1}^{N_{r}}$ for the space $\mathcal{P}_{r}(T)$, the interpolation problem is well-defined if the $N_{r} \times N_{r}$ generalized Vandermonde matrix $V_{\mathcal{B}, X_{r}}$ with entries $\left(V_{X_{r}, \mathcal{B}}\right)_{i j}=\psi_{j}\left(\mathbf{x}_{i}\right), i, j=1, \ldots, N_{r}$, is invertible. The name of Vandermonde matrix is given to $V_{X_{r}, \mathcal{B}}$ in recognition of the fact that, for $d=1$, if the chosen basis is the canonical one $\left\{x^{j-1}\right\}_{j=1}^{N_{r}}$, then $\operatorname{det}\left(V_{X_{r}, \mathcal{B}}\right)$ coincides with the

40 Vandermonde determinant $\Pi_{1 \leq j<i \leq N_{r}}\left(x_{i}-x_{j}\right)$. The solution $\mathcal{I}_{r} f$ of the interpolation problem (1) can be written in terms of the basis functions in $\mathcal{B}$ as $\mathcal{I}_{r} f(\mathbf{x})=\sum_{j=1}^{N_{r}} c_{j} \psi_{j}(\mathbf{x})$, for $\mathbf{x} \in T$, where the vector $\mathbf{c}=\left(c_{1}, \ldots, c_{N_{r}}\right)^{\top}$ is the solution of the linear algebraic system

$$
V_{X_{r}, \mathcal{B}} \mathbf{c}=\mathbf{f}, \quad \mathbf{f}=\left(f\left(\mathbf{x}_{1}\right), \ldots, f\left(\mathbf{x}_{N_{r}}\right)\right)^{\top} .
$$

Inverting the matrix $V_{X_{r}, \mathcal{B}}$ is necessary to compute the dual basis $\left\{\phi_{j}^{X_{r}}\right\}_{j=1}^{N_{r}} \subset$

${ }_{45} \mathcal{P}_{r}(T)$ such that $\phi_{j}^{X_{r}}\left(\mathbf{x}_{i}\right)=\delta_{i j}$, for $1 \leq i, j \leq N_{r}$, being $\delta_{\text {.. the Kronecker's }}$ symbol. The set of points $X_{r}(T)=\left\{\mathbf{x}_{1}, \ldots, \mathbf{x}_{N_{r}}\right\}$ is called unisolvent for the space $\mathcal{P}_{r}(T)$ if the Lagrange interpolation problem (1) is well-defined, namely if for a given basis $\mathcal{B}$ of $\mathcal{P}_{r}(T)$ the Vadermonde matrix $V_{X_{r}, \mathcal{B}}$ is invertible.

Definition 1. Given a d-simplex $T$ and a set $X_{r}(T)$ of $N_{r}$ points in $T$, uni50 solvent for the space $\mathcal{P}_{r}(T)$, the Lebesgue's function $\mathcal{L}_{X_{r}}: T \rightarrow \mathbb{R}^{+}$is defined as

$$
\mathcal{L}_{X_{r}}(\mathbf{x})=\sum_{j=1}^{N_{r}}\left|\phi_{j}^{X_{r}}(\mathbf{x})\right|
$$

with $\left\{\phi_{j}^{X_{r}}\right\}_{j=1}^{N_{r}} \subset \mathcal{P}_{r}(T)$ the Lagrange basis associated with $X_{r}(T)$. The Lebesgue's constant in $T$ is denoted by $\Lambda_{X_{r}}(T)$ and defined as the maximum of the Lebesgue's function $\mathcal{L}_{X_{r}}(\cdot)$ over $T$, namely

$$
\Lambda_{X_{r}}(T)=\left\|\mathcal{L}_{X_{r}}\right\|_{C^{0}(T)}:=\max _{\mathbf{x} \in T}\left|\mathcal{L}_{X_{r}}(\mathbf{x})\right| .
$$


To ensure the stability of the interpolating problem when increasing the polynomial degree, it is crucial to keep under control the growth of $\Lambda_{X_{r}}(T)$ with respect to $r$. The Lebesgue's constant appears in the comparison between the interpolation error $\left\|f-\mathcal{I}_{r} f\right\|_{C^{0}(T)}$ in the maximum norm and the best-fit error $\left\|f-f_{r}^{*}\right\|_{C^{0}(T)}$ in the same norm. Indeed, for any function $f \in C^{0}(T)$, we have

$$
\left\|f-\mathcal{I}_{r} f\right\|_{C^{0}(T)} \leq\left(1+\Lambda_{X_{r}}\right)\left\|f-f_{r}^{*}\right\|_{C^{0}(T)}
$$

where $f_{r}^{*}$ is the best-fit polynomial of degree $r$ of the function $f$ with respect to the maximum norm in $T$, namely, $f_{r}^{*} \in \mathcal{P}_{r}(T)$ and $\left\|f-f_{r}^{*}\right\|_{C^{0}(T)} \leq$ $\|f-p\|_{C^{0}(T)}$ for all $p \in \mathcal{P}_{r}(T)$, so $\left\|f-f_{r}^{*}\right\|_{C^{0}(T)}=\inf _{p \in \mathcal{P}_{r}(T)}\|f-p\|_{C^{0}(T)}=$ $\inf _{p \in \mathcal{P}_{r}(T)} \max _{\mathbf{x} \in T}|f(\mathbf{x})-p(\mathbf{x})|$. The magnitude of the Lebesgue's constant

${ }_{65} \Lambda_{X_{r}}(T)$ depends heavily on the distribution of the points $\mathbf{x}_{i} \in X[7]$. In the bound (3), the growth of the Lebesgue's constant $\Lambda_{X_{r}}$ determines the convergence in the maximum norm. Indeed, we have the indeterminate form $\lim _{r \rightarrow+\infty}\left(\left(1+\Lambda_{X_{r}}\right)\left\|f-f_{r}^{*}\right\|_{C^{0}(T)}\right)=\infty \cdot 0$. If $\Lambda_{X_{r}}$ grows faster in $r$ than the best-fit error dies away, convergence in $r$ is impossible to attain. The inde-

70 termination is solved when it grows up slowly with respect to the interpolation degree $r$, that is for example when it satisfies the condition $\lim _{r \rightarrow+\infty} \Lambda_{X_{r}}^{1 / r}=1$, as proved in [3]. Moreover, the inequality (3) suggests that, when the Lebesgue's constant does not grow too fast, we can find an approximation of a function $f$ on $T$ that is almost as good as the best-fit $f_{r}^{*}$, by just taking the polynomial 75 interpolant $\mathcal{I}_{r} f$, which is generally much easier to compute than $f_{r}^{*}$.

\section{Whitney's forms}

For any integer $k$, with $0 \leq k \leq d$ a $k$-simplex $\sigma$ is the convex hull of $k+1$ linear independent vectors or vertices in $\mathbb{R}^{d}$, with orientation induced by the ordering of the vertices $\sigma=\left[n_{0}, \ldots, n_{k}\right]$. The mass $|\sigma|_{0}$ of a $k$-simplex $\sigma$ is its

so $k$-dimensional Hausdorff's measure. (In particular the mass of any 0-simplex is 1.) A simplicial $k$-chain $c$ is a formal (finite) sum of $k$-simplices with real coefficients. The mass $|c|_{0}$ of a simplicial chain $c=\sum_{i=1}^{I} a_{i} \sigma_{i}$ is defined as $|c|_{0}=\sum_{i=1}^{I}\left|a_{i}\right|\left|\sigma_{i}\right|_{0}$. We denote by $\mathcal{C}_{k}$ (resp. $\left.\mathcal{C}_{k}(T)\right)$ the space of simplicial $k$-chains in $\mathbb{R}^{n}$ (resp. supported in $T$ ).

85 We denote $\Delta_{k}(T)$ the set of $k$-subsimplices of $T$. If $d=3$ then $\Delta_{0}(T)$ is the set of the four vertices of $T, \Delta_{1}(T)$ of its edges, $\Delta_{2}(T)$ of its faces, and $\Delta_{3}(T) \equiv T$. Note that we will consider also other $k$-simplices supported in $T$.

Whitney's $k$-forms of degree one [9] are associated with the $k$-subsimplices of $T$. For each $n \in \Delta_{0}(T)$ the Whitney's 0 -forms $w^{n}$ is the barycentric func-

90 tion $\lambda_{n}$ associated with $n$. For each $\sigma=\left[n_{0}, \ldots, n_{k}\right] \in \Delta_{k}(T)$ we have $w^{\sigma}=$ $\sum_{j=0}^{k}(-1)^{j} \lambda_{n_{j}} \mathrm{~d} w^{\sigma-n_{j}}$ being $w^{\sigma-n_{j}}$ the Whitney's $(k-1)$-form associated with the $(k-1)$-subsimplex of $T$ obtained by eliminating the vertex $n_{j}$ from $\sigma$, and $\mathrm{d}$ the exterior derivative. In particular $w^{T}=|T|_{0}^{-1}$. Whitney's $k$-forms are element of the dual basis for the weights on the $k$-subsimplices of $T$, namely,

${ }_{95} \int_{\sigma^{\prime}} w^{\sigma}=\delta_{\sigma, \sigma^{\prime}}$ for $\sigma, \sigma^{\prime} \in \Delta_{k}(T)$. Note that Whitney's $k$-forms have proxies with components given by polynomials of degree 1 if $0 \leq k<d$ and 0 if $k=d$. 
Whitney's $k$-forms of degree $r>1[8]$ are the elements of the space

$$
\mathcal{P}_{r}^{-} \lambda^{k}(T)=\operatorname{Span}\left\{\lambda^{\alpha} w^{s}: s \in \Delta_{k}(T) \text { and } \alpha \in \mathcal{I}(d, r-1)\right\}
$$

being $\mathcal{I}(d, r-1)=\left\{\left(\alpha_{0}, \ldots, \alpha_{d}\right), \alpha_{i} \in \mathbb{N}, \sum_{i} \alpha_{i}=r-1\right\}$ and $\lambda^{\alpha}=\lambda_{0}^{\alpha_{0}} \ldots \lambda_{d}^{\alpha_{d}}$. We denote $C^{0} \Lambda^{k}\left(\mathbb{R}^{d}\right)$ the Banach's space of continuous $k$-differential forms $w$ with bounded $C^{0}$ norm: $\|w\|_{C^{0}}<\infty$ and $C^{0} \Lambda^{k}(T)$ the space of restrictions of $C^{0} \Lambda^{k}\left(\mathbb{R}^{d}\right)$ to $T$. The norm $\|w\|_{C^{0}}$ has to be intended as the maximum norm of the proxy of $w$.

\section{Polynomial interpolation of forms}

Given a set $X_{k, r}=\left\{s_{1}, \ldots, s_{N_{k, r}}\right\}$ of $N_{k, r}=\operatorname{dim}\left(\mathcal{P}_{r}^{-} \Lambda^{k}(T)\right)$ distint $k$-simplices in $T$ (not necessarily subsimplices of $T$ ), the interpolation problem for a given differential $k$-form $w \in C^{0} \Lambda^{k}(T)$ consists in finding a Whitney's differential $k$-form, $\mathcal{I}_{r} w \in \mathcal{P}_{r}^{-} \Lambda_{k}(T)$, such that $\int_{s_{i}} \mathcal{I}_{r} w=\int_{s_{i}} w$ for all $s_{i} \in X_{k, r}$. As in the case of Lagrange's interpolation, chosen a basis $\mathcal{B}=\left\{w_{j}\right\}_{j=1}^{N_{k, r}}$ for the space $\mathcal{P}_{r}^{-} \Lambda^{k}(T)$ the interpolation problem is well-defined if the generalized Vandermonde's matrix $V_{X_{k, r}, \mathcal{B}}$ with entries $\left(V_{X_{k, r}, \mathcal{B}}\right)_{i, j}=\int_{s_{i}} w_{j}, i, j=1, \ldots, N_{k, r}$, is invertible. We say that the set $X_{k, r}$ of $N_{k, r} k$-simplices supported in $T$ is unisolvent for the space $\mathcal{P}_{r}^{-} \Lambda^{k}\left(\mathbb{R}^{n}\right)$ if the interpolation problem is well-defined.

With any unisolvent set $X_{k, r}$, it is possible to associated a dual basis $\left\{\mathrm{w}_{j}^{X_{k, r}}\right\}_{j=1}^{N_{k, r}}$ of $\mathcal{P}_{r}^{-} \Lambda^{k}\left(\mathbb{R}^{n}\right)$ (namely, such that $\int_{s_{i}} \mathrm{w}_{j}^{X_{k, r}}=\delta_{i, j}$ ). Examples of unisolvent sets of $k$-simplices for the space $\mathcal{P}_{r}^{-} \Lambda^{k}\left(\mathbb{R}^{n}\right)$ are given in [8] and [1].

Definition 2. Given a set $X_{k, r}=\left\{s_{1}, \ldots, s_{N_{k, r}}\right\}$ of $N_{k, r} k$-simplices supported in the $d$-simplex $T$ that is unisolvent for the space $\mathcal{P}_{r}^{-} \Lambda^{k}(T)$, the Lebesgue's function $\mathcal{L}_{X_{k, r}(T)}: \mathcal{C}_{k}(T) \rightarrow \mathbb{R}^{+}$is defined as

$$
\mathcal{L}_{X_{k, r}}^{k}(c)=\sum_{j=1}^{N_{k, r}}\left|s_{j}\right|_{0}\left|\int_{c} \mathrm{w}_{j}^{X_{k, r}}\right|
$$

with $\left\{\mathrm{w}_{j}^{X, r}\right\}_{j=1}^{N_{k, r}} \subset \mathcal{P}_{r}^{-} \Lambda^{k}(T)$ the dual basis associated with $X_{k, r}$. The Lebesgue's 120 constant is then $\Lambda_{X_{k, r}}^{k}=\sup _{c \in \mathcal{C}_{k}(T)} \frac{\mathcal{L}_{X_{k, r}}^{k}(c)}{|c|_{0}}$.

Proposition 1. If $k=0$ then Definition 2 coincides with Definition 1 .

Proof: To prove that $\Lambda_{X_{0, r}} \leq \Lambda_{X_{0, r}}^{0}$, we recall that the 0-simplices supported in $T$ are points $\mathbf{x}$ of $T,|\mathbf{x}|_{0}=1$, and $\phi_{j}^{X_{0, r}}(\mathbf{x})=\int_{\mathbf{x}} \phi_{j}^{X_{0, r}}$. Being $\Lambda_{X_{0, r}}=$ $\max _{\mathbf{x} \in T} \sum_{j=1}^{N_{0, r}}\left|\phi_{j}^{X_{0, r}}(\mathbf{x})\right|$, we have

$$
\Lambda_{X_{0, r}}=\max _{\mathbf{x} \in T} \frac{1}{|\mathbf{x}|_{0}} \sum_{j=1}^{N_{0, r}}\left|\phi_{j}^{X_{0, r}}(\mathbf{x})\right|=\max _{\mathbf{x} \in T} \frac{1}{|\mathbf{x}|_{0}} \sum_{j=1}^{N_{0, r}}\left|\int_{\mathbf{x}} \phi_{j}^{X_{0, r}}\right|
$$




$$
\Lambda_{X_{0, r}} \leq \sup _{c \in \mathcal{C}_{0}(T)} \frac{1}{|c|_{0}} \sum_{j=1}^{N_{0, r}}\left|\int_{c} \phi_{j}^{X_{0, r}}\right|=\sup _{c \in \mathcal{C}_{0}(T)} \frac{\mathcal{L}_{X_{0, r}}^{0}(c)}{|c|_{0}}=\Lambda_{X_{0, r}}^{0},
$$

where we have used that $\phi_{j}^{X_{0, r}}=\mathrm{w}_{j}^{X_{0, r}}$.

On the other hand, if $c=\sum_{i=1}^{I} a_{i} \mathbf{x}_{i} \in \mathcal{C}_{0}(T)$ then, taking into account that $\left|s_{i}\right|_{0}=1$ for each $s_{i} \in X_{0, r}$, one has

$$
\begin{aligned}
\mathcal{L}_{X_{0, r}}^{0}(c) & =\sum_{j=1}^{N_{0, r}}\left|\int_{c} \phi_{j}^{X_{0, r}}\right|=\sum_{j=1}^{N_{0, r}}\left|\sum_{i=1}^{I} a_{i} \phi_{j}^{X_{0, r}}\left(\mathbf{x}_{i}\right)\right| \\
& \leq \sum_{j=1}^{N_{0, r}} \sum_{i=1}^{I}\left(\left|a_{i}\right|\left|\phi_{j}^{X_{0, r}}\left(\mathbf{x}_{i}\right)\right|\right)=\sum_{i=1}^{I}\left(\left|a_{i}\right| \sum_{j=1}^{N_{0, r}}\left|\phi_{j}^{X_{0, r}}\left(\mathbf{x}_{i}\right)\right|\right) \\
& \leq \sum_{i=1}^{I}\left(\left|a_{i}\right| \max _{\mathbf{x} \in T} \sum_{j=1}^{N_{0, r}}\left|\phi_{j}^{X_{0, r}}(\mathbf{x})\right|\right)=|c|_{0} \Lambda_{X_{r}},
\end{aligned}
$$

because for a chain $c=\sum_{i=1}^{I} a_{i} \mathbf{x}_{i} \in \mathcal{C}_{0}(T)$ one has $|c|_{0}=\sum_{i=1}^{I}\left|a_{i}\right|$. Therefore $\Lambda_{X_{0, r}}^{0}=\sup _{c \in \mathcal{C}_{0}(T)} \frac{\mathcal{L}_{X_{0, r}(c)}^{0}}{|c|_{0}} \leq \Lambda_{X_{0, r}}$.

For any $L^{1}$-differential $k$-form $w$ we define $\|w\|_{0}:=\sup _{\sigma \neq 0} \frac{\left|\int_{\sigma} w\right|}{|\sigma|_{0}}$, where the supremum is taken over all $k$-subsimplices of $T$. If $w \in C^{0} \Lambda^{k}(T)$ then $\|w\|_{0}=\|w\|_{C^{0}}$. (See, e.g., [].)

Proposition 2. Let $X_{k, r}(T)=\left\{s_{1}, \ldots, s_{N_{k, r}}\right\}$ be a unisolvent set of $N_{k, r} k$ simplices supported in the $d$-simplex $T$ that is unisolvent for the space $\mathcal{P}_{r}^{-} \Lambda^{k}(T)$. For each differential $k$-form $w \in C^{0} \Lambda^{k}(T)$ it holds

$$
\left\|w-\mathcal{I}_{r} w\right\|_{0} \leq\left(1+\Lambda_{X_{k, r}}\right)\left\|w-w^{*}\right\|_{0}
$$

where $w^{*}$ is the best-fit reconstruction of $w$ for the given norm $\|\cdot\|_{0}$, namely $w^{*} \in \mathcal{P}_{r}^{-} \Lambda^{k}(T)$ and it verifies $\left\|w-w^{*}\right\|_{0} \leq\|w-z\|_{0}$ for all $z \in \mathcal{P}_{r}^{-} \Lambda^{k}(T)$.

Proof: First we notice that $\mathcal{I}_{r} w^{*}=w^{*}$ and

$$
\left\|w-\mathcal{I}_{r} w\right\|_{0} \leq\left\|w-w^{*}\right\|_{0}+\left\|\mathcal{I}_{r}\left(w^{*}-w\right)\right\|_{0} \leq\left(1+\left\|\mathcal{I}_{r}\right\|_{0}\right)\left\|w-w^{*}\right\|_{0}
$$

${ }_{140}$ being $\left\|\mathcal{I}_{r}\right\|_{0}:=\sup _{w \in C^{0} \Lambda^{k}(T)} \frac{\left\|\mathcal{I}_{r} w\right\|_{0}}{\|w\|_{0}}$. Recalling that $\mathcal{I}_{r} w=\sum_{j=1}^{N_{k, r}}\left(\int_{s_{j}} w\right) \mathrm{w}_{j} X_{k, r}$ we have

$$
\begin{aligned}
\left|\int_{c} \mathcal{I}_{r} w\right| & =\left|\sum_{j=1}^{N_{k, r}} \int_{s_{j}} w \int_{c} \mathrm{w}_{j} X_{k, r}\right| \leq \sum_{j=1}^{N_{k, r}} \frac{\left|\int_{s_{j}} w\right|}{\left|s_{j}\right|_{0}}\left|s_{j}\right|_{0}\left|\int_{c} \mathrm{w}_{j}^{X}\right| \\
& \leq \max _{1 \leq j \leq N_{k, r}} \frac{\left|\int_{s_{j}} w\right|}{\left|s_{j}\right|_{0}} \sum_{j=1}^{N_{k, r}}\left|s_{j}\right|_{0}\left|\int_{c} \mathrm{w}_{j} X_{k, r}\right| \leq\|w\|_{0} \mathcal{L}_{X_{k, r}}^{k}(c) .
\end{aligned}
$$

It is easy to check that $\|w\|_{0}=\sup _{c \in \mathcal{C}_{k}} \frac{\left|\int_{c} w\right|}{|c|_{0}}$, so

$$
\left\|\mathcal{I}_{r} w\right\|_{0}=\sup _{c \in \mathcal{C}_{k}} \frac{\left|\int_{c} \mathcal{I}_{r} w\right|}{|c|_{0}} \leq\|w\|_{0} \sup _{c \in \mathcal{C}_{k}} \frac{\mathcal{L}_{X_{k, r}}^{k}(c)}{|c|_{0}}=\|w\|_{0} \Lambda_{X_{k, r}}^{k}
$$

and $\left\|\mathcal{I}_{r}\right\|_{0}:=\sup _{w \in C^{0} \Lambda^{k}(T)} \frac{\left\|\mathcal{I}_{r} w\right\|_{0}}{\|w\|_{0}} \leq \Lambda_{X_{k, r}}^{k}$. Replacing in 5 we obtain 44. 


\section{Some remarks and conclusions}

We have generalized the definition of the Lebesgue's function and constant 145 to the case of $k$-form interpolation in $\mathcal{P}_{r}^{-} \Lambda^{k}$, the space of "trimmed" polynomial differential forms widely used in the finite elements exterior calculus (see [2]). This extension is very natural when using the weights on subsimplices as degrees of freedom due to their clear physical meaning. For $r>1$, the subsimplices have to be viewed as an enrichment of the geometrical description of the weights' support, as if we were computing a more accurate approximation of circulations $(\mathrm{k}=1)$, fluxes $(\mathrm{k}=2)$, etc.

As in the classical setting of Lagrange's polynomial interpolation (that in fact correponds to the case $k=0$ ), the Lebesgue's constant is a quality indicator when selecting among unisolvent sets of degrees of freedom, the ones that

155 minimize the local interpolation error in the maximum norm. Applications are postponed to future works.

\section{References}

[1] Alonso Rodríguez, A., Bruni Bruno, L., Rapetti, F.: Minimal sets of unisolvent weights for high order Whitney forms on simplices. Enumath 2019 procs., LNCSE 139 (2020) in press.

[2] Arnold, D. N., Falk, R. S., Ragnar Winther, R.: Finite element exterior calculus, homological techniques, and applications. Acta Numerica, 15 (2006), $1-155$.

[3] Bloom, T.: The Lebesgue constant for Lagrange interpolation in the simplex. Journal of the Atmospheric Sciences, 54 (1988), 338-353.

[4] Bossavit, A.: Computational Electromagnetism, New York, NY, USA: Academic, 1998.

[5] Chung, K.C., Yao, T.H.: On lattices admitting unique Lagrange interpolations. SIAM J. Numer. Anal., 14, 735-743 (1977).

[6] Harrison, J.: Continuity of the integral as a function of the domain. J. Geom. Anal. 8 (1998) 769-795.

[7] Pasquetti, R., Rapetti, F.: Spectral element methods on unstructured meshes: Which interpolation points ?, Numerical Algorithms, 55/2 (2010) 349-366.

[8] Rapetti, F., Bossavit, A.: Whitney forms of higher degree, SIAM J. Numer. Anal., 47/3 (2009) 2369-2386.

[9] Whitney, H.: Geometric Integration Theory, Princeton, NJ, USA: Princeton Univ. Press, 1957. 